\title{
Prospects for SUSY searches in CMS and ATLAS
}

\author{
Paul de Jong, on behalf of the CMS and ATLAS collaborations
}

Nikhef, P.O. Box 41882, NL-1009 DB Amsterdam, the Netherlands

\begin{abstract}
We discuss how the CMS and ATLAS experiments are preparing for the analysis of first LHC data with emphasis on the search for supersymmetry. We will show the importance of the understanding of detector, trigger, reconstruction and backgrounds, and we will present realistic estimates of the reach of CMS and ATLAS.
\end{abstract}

Keywords: supersymmetry, LHC, CMS, ATLAS

PACS: 12.60.Jv, 14.80.Ly

\section{INTRODUCTION}

After a run at ${ }^{\mathrm{P}} \overline{\mathrm{s}}=10 \mathrm{TeV}$ in 2008, the Large Hadron Collider (LHC) at CERN is likely to deliver a few $\mathrm{fb}^{1}$ of integrated luminosity at $14 \mathrm{TeV}$ in 2009 , and keep increasing its luminosity in further years. The CMS and ATLAS experiments are general multipurpose detectors designed to analyze the results of the collisions. Due to the high centre-of-mass energy and the high luminosity of the LHC, the prospects for searches for new physics beyond the Standard Model (SM), including supersymmetry (SUSY) are excellent.

At the time of writing of these proceedings, first beams have been injected in the LHC. Both experiments are basically ready for beam; in the last year(s) both CMS [1] and ATLAS [2] have actively prepared for SUSY searches. For lack of space, only a selection of results is shown. Since at the time of writing there is no LHC data yet, all "results" have been obtained with realistic Monte Carlo simulations. Although this note focuses on searches for supersymmetry, CMS and ATLAS perform many more searches for general new physics beyond the SM.

\section{CONFIDENCE BUILDING}

Searching for SUSY equals confidence building. Only with confidence in the operation and performance of the detector, in the trigger, in the reconstruction and object identification methods, and in our knowledge of the backgrounds, can we claim to see excesses over the SM and evidence for new physics. This confidence building will be the primary activity of the experiments with first data, and it will need to continue thereafter. Although with a very small data sample we are in principle sensitive to SUSY beyond the Tevatron, confidence building needs time, hard work and luminosity.

The primary objective of the experiments now is to establish reliable, long-term, controlled and safe running of the detectors. With systems as complex as those of CMS and ATLAS, this is not a triviality. During this process, we will need to get to know the 

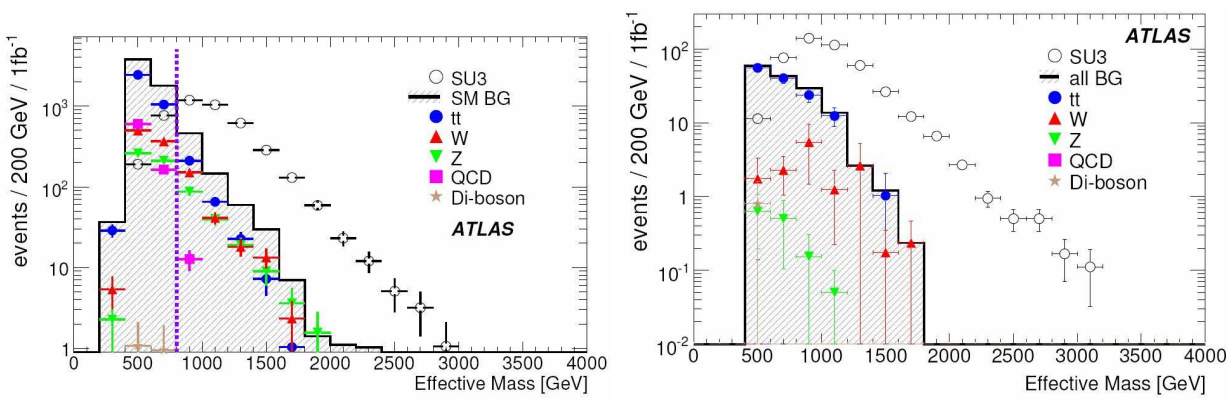

FIGURE 1. Backgrounds in the no-lepton (left) and in the one-lepton (right) search modes, for ATLAS in $1 \mathrm{fb}^{1}$, after preselection cuts requiring at least four energetic jets and significant missing transverse energy. Also the SUSY signal of the SU3 benchmark point is shown.

detectors like the back of our hand: their problematic regions, dead or noisy cells, etc.

Triggering on SUSY should not be difficult: there are likely to be energetic jets, significant missing transverse energy, electrons, muons, taus, photons, and/or b-quark jets. In combination, trigger efficiencies of better than $95 \%$ should be attainable. However, the trigger performance should be demonstrated from the data, and the trigger should be designed with this in mind. Also background control samples should be triggered on.

Confidence in the reconstruction and object (electrons, muons, jets etc.) identification will be gained from detailed studies of first data. Calibration- and alignment constants must be derived and applied, and energy- and momentum scales set. In $1 \mathrm{pb}^{1}$ (3 days at $10^{31} \mathrm{~cm}^{2} \mathrm{~s}^{1}$ ), $16000 J=\Psi ! \mu^{+} \mu$ and $3000 \Upsilon ! \mu^{+} \mu$ events are expected; in 10 $\mathrm{pb}{ }^{1}$ some $6000 \mathrm{Z}$ ! $\mu^{+} \mu$ and a simular number to $e^{+} e$ should be collected. From these relatively simple topologies we move to more complex ones by allowing additional jets; when we approach $100 \mathrm{pb}^{1}$ the top quark becomes an excellent calibration tool. Clean $t \bar{t}$ samples can be selected and used to verify lepton identification, jet- and missing transverse energy scales, and b-quark tagging.

\section{BACKGROUNDS}

Confidence in the understanding of the SM backgrounds can and will be gained by a dedicated campaign that involves data-driven background determinations, aided by Monte Carlo wherever needed [3]. The validation of Monte Carlo's with data is also an early goal. A number of background estimation methods are under development in CMS and ATLAS; only their consistency in combination can lead to the desired confidence in understanding. Even then, statistical and systematic uncertainties on the backgrounds remain, which must be taken into account in the SUSY searches.

After demanding a few energetic jets and some missing transverse energy $E_{T}^{\text {miss }}$, as one would do in a preselection for a typical R-parity conserving SUSY analysis, the SM background consists largely of QCD multi-jet production, $\mathrm{W}$ and $\mathrm{Z}$ production with extra jets, top-pair production, and to a lesser extent di-boson and single top production, as shown in Figure 1 . 

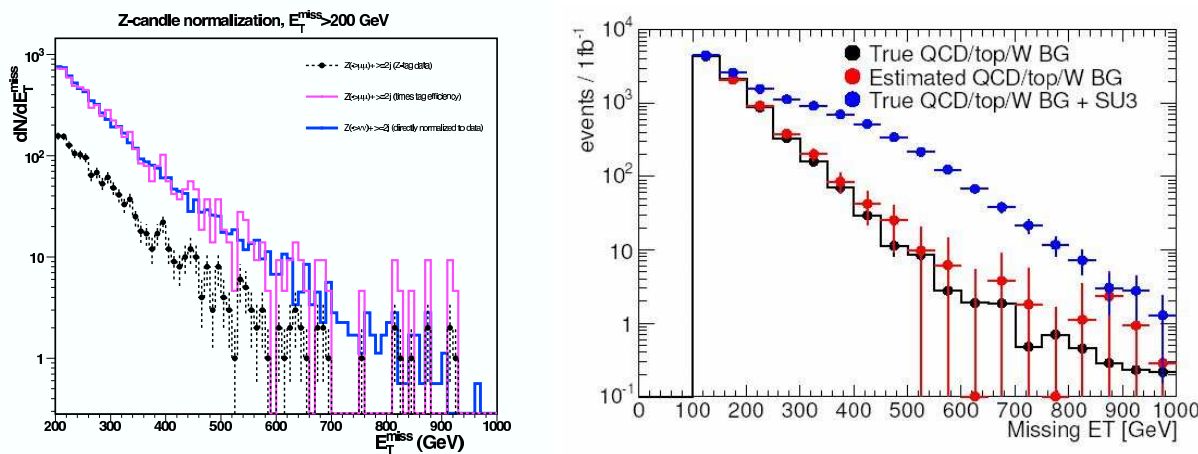

FIGURE 2. Data-driven methods for background estimation. Left: CMS estimate of $\mathrm{Z}(! \quad v v)+$ jets background from a $\mathrm{Z}\left(! \mu^{+} \mu\right)$ +jets control sample. Right: ATLAS estimate of QCD + top + W background in the no-lepton mode, compared to the "true" background from Monte Carlo, and the SU3 SUSY signal.

\section{No-lepton final state}

In this search mode, with a veto on isolated leptons, QCD multi-jet production in principle dominates the background. $E_{T}^{\text {miss }}$ can be generated by neutrinos from heavy quark decay, or is "fake", i.e. generated by detector effects or backgrounds unrelated to the collision such as cosmic rays or beam halo. Clean-up cuts such as calorimeter timing cuts, a good primary vertex, and jet shower shape cuts are needed. Mis-measured jets can lead to fake $E_{T}^{\text {miss }}$; in this case the $E_{T}^{\text {miss }}$ direction will point to one of the jets, and such topologies can be cut away by demanding isolation of the $E_{T}^{\text {miss }}$ vector. The remaining $E_{T}^{\text {miss }}$ in the QCD multi-jet background should be determined from data. This can be done using prescaled jet triggers, or with clean samples of top-quark pairs and Z+jets events. ATLAS has also studied a method that involves measuring an $E_{T}^{\text {miss }}$ response function from data (the Gaussian part from photon+jets events, the tails from a sample of three-jet events with the missing momentum vector pointing towards or away from one of the jets), and applying this function to a large sample of balanced, low $E_{T}^{\text {miss }}$, events.

Further backgrounds in the no-lepton mode include $\mathrm{Z}+\mathrm{jets}$ events with the $\mathrm{Z}$ decaying to neutrinos, top-quark pair events and $\mathrm{W}+$ jets events. The $\mathrm{Z}$ ! $v v$ background can be effectively estimated from $Z ! \mu^{+} \mu$ control samples, as shown in Figure 2 (left). The top and $\mathrm{W}$ backgrounds are most dangerous when the $\mathrm{W}$ decays into a lepton and a neutrino, but the lepton is not identified, for example because it is a tau lepton, or falls outside the acceptance. Various methods to estimate this from data are being developed, Figure 2 (right) shows an example.

\section{Final state with one lepton}

In the one-lepton search mode, the presence of a high $p_{T}$ isolated electron or muon is required. This facilitates triggering on the event and suppresses the QCD background, 
TABLE 1. Expected statistical and systematic uncertainties on the background, as expected by ATLAS in $1 \mathrm{fb}^{1}$. The top rows apply to the no-lepton mode, the bottom rows to the one-lepton mode, and $t \bar{t}$ and $\mathrm{W}+$ jets apply to both modes.

\begin{tabular}{|c|c|c|}
\hline Source & Stat. unc. (\%) & Syst. unc. (\%) \\
\hline $\begin{array}{l}\text { QCD multi-jets } \\
\text { top } ! \tau\end{array}$ & $\begin{array}{l}1 \\
6\end{array}$ & $\begin{array}{l}50 \\
15\end{array}$ \\
\hline $\mathrm{Z} ! \quad v \bar{v}$ & $8 \quad 13$ & $10 \quad 15$ \\
\hline$t \bar{t}$ and $\mathrm{W}+$ jets & 48 & 15 \\
\hline $\begin{array}{l}t \bar{t} \text { semi-leptonic } \\
t \bar{t} \text { di-leptonic }\end{array}$ & $\begin{array}{c}5 \\
10\end{array}$ & $\begin{array}{l}22 \\
20\end{array}$ \\
\hline
\end{tabular}

but also costs signal efficiency. Nevertheless, the one-lepton mode is a robust way to look for SUSY and will play an important role. Figure 1 (right) shows the expected backgrounds for the one-lepton mode after preselection cuts. It is dominated by topquark pairs and $\mathrm{W}+\mathrm{jets}$ events; a sizable fraction of the top background comes from dileptonic top events with one lepton not identified. Details of data-driven estimation methods are described elsewhere in these proceedings [3].

\section{Uncertainties on the background}

Table 1 lists the uncertainties on the background as currently expected by ATLAS with data-driven techniques, in $1 \mathrm{fb}^{1}$. The statistical uncertainties apply to the ATLAS cuts before the final $M_{\text {eff }}$ cut.

\section{MODELS AND BENCHMARKS}

Obviously, the main objective for CMS and ATLAS in searches for new physics is: don't miss it [4]. Therefore, signatures and topologies must be covered as complete and as general as possible. The searches should be kept robust and inclusive, in combination with exclusive measurements making use of specific supersymmetry-related signatures.

The baseline searches are done assuming R-parity conservation, where production and decay of squarks and gluinos lead to energetic jets, $E_{T}^{\text {miss }}$ from the unobserved lightest supersymmetric particle, and possibly leptons. R-parity violating searches are also performed, but are not covered here. The interpretation of results is typically performed in the mSUGRA framework, but also in the NUHM, GMSB and AMSB frameworks.

In order to study selection cuts, use is made of a number of SUSY benchmark points with a specific choice of model parameters. Most important is the coverage of signatures and a good representation of phase space; the exact details of each point are not very relevant. CMS and ATLAS have each chosen their own set of benchmark points, for 
TABLE 2. A rough comparison of mSUGRA benchmark points in use by CMS and ATLAS, and the Snowmass [5] and BDEGMOPW [6] points.

\begin{tabular}{|llll|l|}
\hline Snowmass & CMS & ATLAS & BDEGMOPW & Description \\
\hline SPS 1a' & LM1 & SU3 & B' & "bulk" \\
SPS 1b & & SU8.1 & & "high $\tan \beta$ bulk" \\
SPS 2 & LM9 & & E' & "focus point" \\
& LM7 & SU2 & & "high $m_{0}$ focus point" \\
SPS 3 & LM6 & SU1 & C' & "co-annihilation" \\
SPS 4 & LM2 & SU6 & I'/L & "high tan $\beta "$ \\
& & SU4 & & "low mass" \\
\hline
\end{tabular}

ease of comparison Table 2 gives a rough translation of mSUGRA benchmark points in use.

\section{INCLUSIVE SEARCHES}

$$
\text { Jets plus } E_{T}^{\text {miss }} \text { plus } X
$$

In order to study the selection and discovery reach, recent ATLAS studies have assumed a data sample of $1 \mathrm{fb}^{1}$, and a corresponding knowledge of detector-related systematic uncertainties. Furthermore, the expected uncertainties of the background, as derived with data-driven estimation methods discussed earlier, are taken into account. The significance of the signal is then calculated from the probability of the background, including uncertainties, to fluctuate to the signal.

ATLAS makes a baseline selection of at least four energetic jets, significant $E_{T}^{\text {miss }}$, and transverse sphericity $S_{T}>0.2$ [4]. In the no-lepton search mode, no isolated high $p_{T}$ electron or muon is allowed. A discriminating variable between SUSY and background is the effective mass $M_{\text {eff }}$, defined as the sum of the $p_{T}$ of the leading four jets and $E_{T}^{\text {miss }}$. With an additional cut on $M_{\text {eff }}>800 \mathrm{GeV}$, a sensitivity of (significantly) more than $5 \sigma$ is reached for all ATLAS benchmark points except SU2. Analyses optimized for at least two or at least three jets have somewhat better significance, but are more sensitive to the QCD multi-jet background.

In the one-lepton mode, one identified high $p_{T}$ isolated electron or muon is required, and the transverse mass of lepton and $E_{T}^{\text {miss }}$ should be larger than $100 \mathrm{GeV}$. The lepton requirement reduces the signal somewhat, but suppresses QCD background, and again gives excellent sensitivity.

The CMS studies have assumed either 1 or $10 \mathrm{fb}{ }^{1}$, and a corresponding knowledge of detector-related systematic uncertainties. In the no-lepton mode, CMS demands at least three energetic central jets and large $E_{T}^{\text {miss }}$, and vetoes isolated leptons. Finally, $H_{T}>500 \mathrm{GeV}$ is demanded, where $H_{T}$ is the sum of the $E_{T}$ of jets 2,3 and 4 , and $E_{T}^{\text {miss }}$. Similar cuts, but requiring an isolated lepton, are used on the one-lepton mode. Further analyses look for tau leptons, a $\mathrm{Z}$ or a top quark together with jets and $E_{T}^{\text {miss }}$.

The CMS and ATLAS discovery reach for $1 \mathrm{fb}{ }^{1}$, interpreted in the mSUGRA model for $A_{0}=0, \tan \beta=10, \mu>0$, is shown in Figure 3, clearly these sensitivities are 

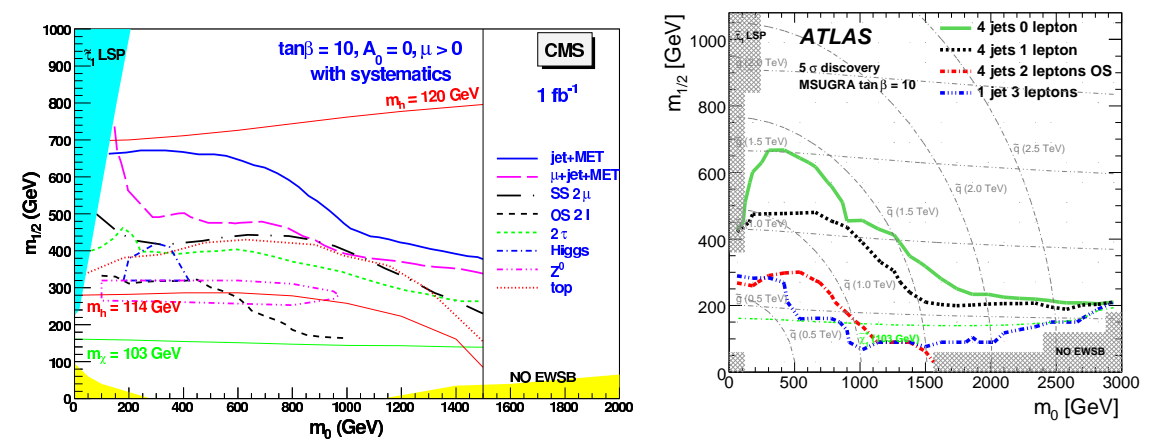

FIGURE 3. Discovery reach of CMS (left) and ATLAS (right) in $m_{1=2}$ and $m_{0}\left(A_{0}=0, \tan \beta=10\right.$, $\mu>0)$ in mSUGRA, for $1 \mathrm{fb}{ }^{1}$.
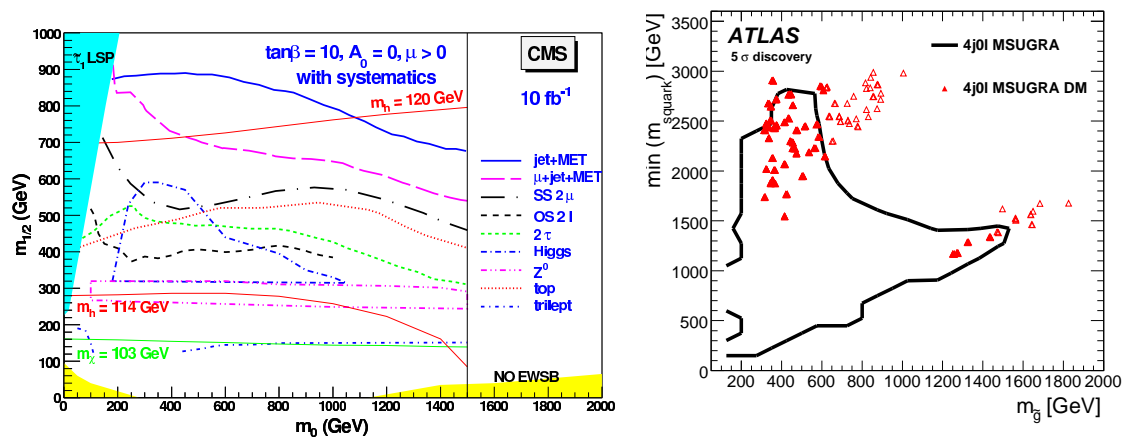

FIGURE 4. Idem for CMS in $10 \mathrm{fb}^{1}$, and for ATLAS in $1 \mathrm{fb}{ }^{1}$ expressed in squark and gluino masses.

comparable. Figure 4 shows the expected CMS reach in $10 \mathrm{fb}^{1}$ and the ATLAS reach for $1 \mathrm{fb}{ }^{1}$ expressed in squark and gluino mass range.

Further interesting search modes include the di-lepton and tri-lepton searches. Demanding the two leptons in the di-lepton mode to have the same charge effectively suppresses background, but leaves a significant SUSY signal [7]. The tri-lepton searches are sensitive to direct chargino/neutralino production, for example in the "focus region", but need significant luminosity $\left(>10 \mathrm{fb}^{1}\right)$ [8]. In these modes, the background can probably be estimated from data with similar methods as those used in the one-lepton mode.

\section{GMSB models}

In models with gauge-mediated SUSY breaking (GMSB), the LSP is a gravitino that can be very light. The next-to-lightest SUSY particle (NLSP) is typically either the lightest neutralino or a stau (or the various sleptons are almost degenerate co-NLSPs). The NLSPs decay into the LSP plus typically a photon, or a lepton (tau). This decay can be prompt, or the NLSP can have a significant lifetime, decaying away from the primary 

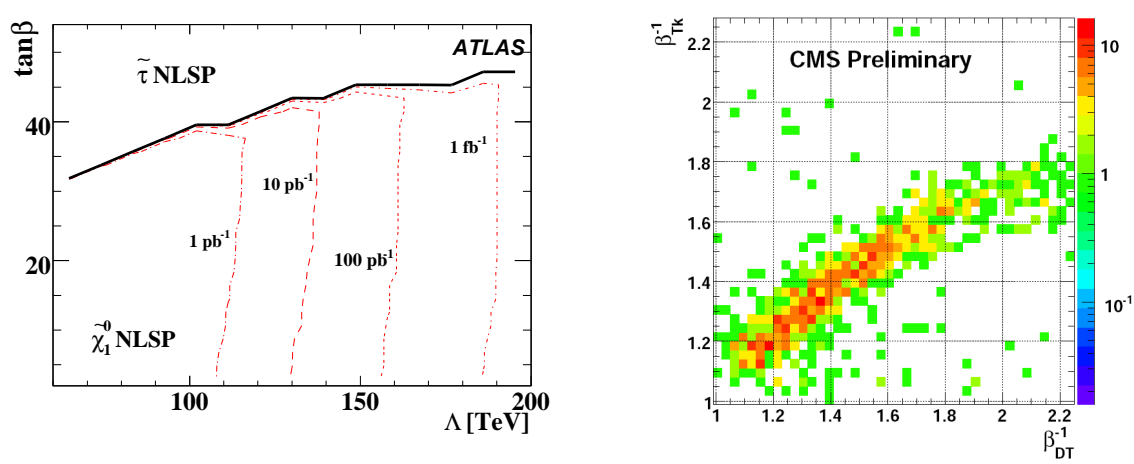

FIGURE 5. Left: ATLAS discovery reach in GMSB parameters $\Lambda$ and $\tan \beta$ in the analysis that searches for isolated high $p_{T}$ photons and $E_{T}^{\text {miss }}$. Right: $1=\beta$ measured from the CMS tracker (Tk) versus $1=\beta$ measured from the CMS muon drift tubes (DT), for a long-living stop quark with a mass of $500 \mathrm{GeV}$. The $\mathrm{SM}$ backgrounds cluster around $1=\beta_{\mathrm{Tk}}=1$ and/or $1=\beta_{\mathrm{DT}}=1$.

vertex or even leaving the detector.

The ATLAS strategy for a search for prompt energetic photons from $\tilde{\chi}_{1}^{0} ! \gamma \tilde{G}$ leads to a sensitivity as shown in Figure 5 (left).

Neutralinos decaying away from the main vertex can lead to photons in the detector that do not point to the main vertex. Special reconstruction techniques must be applied to reconstruct such photons, and both CMS and ATLAS have been studying this.

Massive charged particles with a long lifetime, for example long-living stau leptons or stop quarks, will leave a track in the tracking detectors of CMS or ATLAS. A decay inside the tracking detectors can give rise to tracks with significant kinks. NLSPs with an even longer lifetime leave a track through the whole detector, from tracking detectors to the muon chambers. Such a track is thus reconstructed as a muon; however due to the large NLSP mass its velocity $\beta$ might be significantly smaller than $c$ [9]. Such slow particles may pose a problem to trigger and reconstruction. Both CMS and ATLAS have studied this [10, 2]. Figure 5](right) shows for CMS the measured $1=\beta$ from the tracker versus the measured $1=\beta$ from the muon system for a long-living stop quark with a mass of $500 \mathrm{GeV}$. If both momentum and velocity can be reconstructed, the NLSP mass can be estimated. Figure 6 (left) shows the amount of luminosity needed by CMS to make a $5 \sigma$ discovery of several classes of massive semi-stable charged particles as a function of their mass.

\section{INTERPRETATION}

An inclusive discovery of new physics with jets and $E_{T}^{\text {miss }}$, or with signatures as in the GMSB model, would be spectacular, but would not reveal much of the underlying physics. Several models of new physics can fit the observed data, and certainly the new physics can also be of a kind outside any existing model. Establishing the nature of any observed deviation from the SM demands hard work and ingenuity. Even then, ambiguities may remain.

Interpretation of any excess in terms of SUSY asks for consistency of signals in 

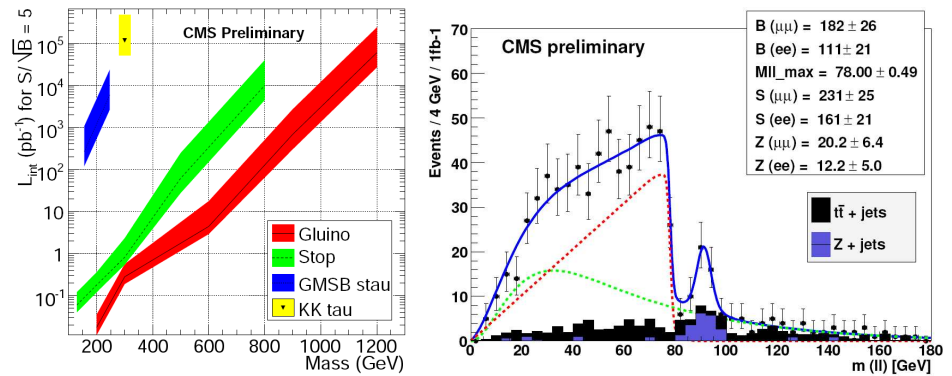

FIGURE 6. Left: luminosity needed by CMS to make a $5 \sigma$ discovery of several classes of massive semi-stable charged particles as a function of their mass. Right: The edge in dilepton mass in the CMS LM1 model, for $1 \mathrm{fb}^{1}$. Red: signal pdf, green: flavour-symmetric background pdf.

various final states, mass scales, branching fractions, cross-sections, and a proof of the spin of the newly observed particles. A first estimate of the mass scale of squarks and gluinos can be derived from the effective mass distribution and from the measured crosssections. More information, however, can be gained from exclusive studies.

In particular, one can try and select a suitable decay chain like

$$
\tilde{q}_{L} ! \tilde{\chi}_{2}^{0} q(! \tilde{\imath}, q) ! \tilde{\chi}_{1}^{0,}, q ;
$$

and measure invariant masses of combinations of objects like the two leptons, the leptons and the jet, or one lepton and the jet. The distributions of these masses typically have thresholds and edges sensitive to the masses of particles in the decay chain. Figure 6 (right) shows a study of the dilepton mass edge by CMS in the LM1 benchmark model, in $1 \mathrm{fb}^{1}[11]$.

ATLAS has studied the precision of parameter extraction in the SU3 and SU4 models with 1 , or $0.5 \mathrm{fb}^{1}$ [12]. Already such limited luminosity will give a first hint of underlying parameters.

A clear hint that SUSY particles are being produced could come from a measurement of particle spin. This is difficult, and has been studied by ATLAS for neutralinos and sleptons [13].

\section{FLAVOUR-ORIENTED STUDIES}

Since in SUSY flavour-universality is very likely broken, it is interesting to perform studies that concentrate on the properties of individual flavours.

A study of the mass distribution of $e \mu$ combinations is sensitive to lepton-flavour violating neutralino decays $\tilde{\chi}_{2}^{0}$ ! $e \quad \mu \quad \tilde{\chi}_{1}^{0}$. A CMS study has shown $5 \sigma$ sensitivity to lepton-flavour violating branching fractions at the $4 \%$ level with $10 \mathrm{fb}{ }^{1}$.

The lightest stop quark, $\tilde{t}_{1}$, is likely to be the lightest squark. CMS has analyzed the potential of a stop quark discovery through its decay $\tilde{t} ! \quad t \tilde{\chi}_{2}^{0} ! t^{{ }^{\prime}}{ }_{R}{ }^{\prime} ! t^{\prime \prime} \tilde{\chi}_{1}^{0}$. In the events, the top quarks are kinematically reconstructed with a kinematic fit. In the LM1 point, $M_{\tilde{t}_{1}} \quad 400 \mathrm{GeV}$, and a $5 \sigma$ discovery is possible with $200 \mathrm{pb}{ }^{1}$. 
ATLAS has studied $\tilde{g} ! \tilde{t} t ! \tilde{\chi} b t$ in the SU4 (low mass) point. In this point, $M_{\tilde{t}_{1}} \quad 200 \mathrm{GeV}$, and it is possible to reconstruct the events and plot the top-bottom mass distribution, which shows an edge sensitive to the stop mass. In a sample of $200 \mathrm{pb}{ }^{1}$, almost 1000 signal events would be observed, against 100 SM background events.

Furthermore, ATLAS has studied a scenario in which the lightest stop is lighter than the top quark. Top-quark pair production is the largest background to this search, but can be subtracted using a side-band technique. Approximately $1 \mathrm{fb}^{1}$ of data should be enough to see a signal.

\section{CONCLUSIONS AND OUTLOOK}

We are at the threshold of exciting times. Some 15 years of planning, designing, construction, and installation of the detectors are coming to an end. With the LHC data we will either discover SUSY, or push its mass scale so high that it is no longer a natural solution to the fine tuning problem. Let the data decide.

Excellent prospects for the SUSY search already exist with little luminosity. However, what CMS and ATLAS really need now is luminosity to shake down the detector, the trigger, the reconstruction, and understanding of the backgrounds. Only data can give

the confidence that is needed to claim evidence for new phenomena. With $1-2 \mathrm{fb}{ }^{1}$ of integrated luminosity (2009?), we will be able to gain that confidence, and we will be sensitive to a very interesting region of SUSY parameter space [14].

\section{ACKNOWLEDGMENTS}

The author wishes to thank everyone in CMS and ATLAS whose work contributed to this talk.

\section{REFERENCES}

1. The CMS Collaboration, CMS Physics TDR, CERN-LHCC-2006-021 (2006).

2. The ATLAS Collaboration, Expected performance of the ATLAS experiment - Detector, Trigger and Physics -, CERN-OPEN-2008-020 (2008)

3. F. Legger, these proceedings.

4. C. Autermann, these proceedings.

5. B. C. Allanach et al., The Snowmass points and slopes: Benchmarks for SUSY searches, hep-ph/0202233 (2002).

6. M. Battaglia et al., Updated post-WMAP benchmarks for supersymmetry, Eur. Phys. J. C33 (2004) 273.

7. Y. Pakhotin, these proceedings.

8. T. Sarangi, these proceedings.

9. D. Teyssier, these proceedings.

10. The CMS Collaboration, CMS PAS EXO-08-003 (2008).

11. The CMS Collaboration, CMS PAS SUS-08-001 (2008).

12. P. Wienemann, these proceedings.

13. M. Biglietti et al, ATLAS Conference Note ATL-PHYS-CONF-2007-020 (2007), ATL-PHYS-PUB2007-004 (2007); A. J. Barr, JHEP 02 (2006) 042.

14. S. Heinemeyer, these proceedings. 